\title{
PROPOSTA METODOLÓGICA PARA O MAPEAMENTO DE DANOS CAUSADOS POR EVENTOS EXTREMOS EM PRAIAS DENSAMENTE URBANIZADAS
}

\author{
Methodological framework for mapping the damages \\ caused by extreme events in densely urbanized beaches
}

Thales Vargas Furtado', Jarbas Bonetti ${ }^{2}$

\begin{abstract}
${ }^{1}$ Programa de Pós-Graduação em Geografia, Universidade Federal de Santa Catarina, ex-bolsista de mestrado da Capes, Laboratório de Oceanografia Costeira, Coordenadoria Especial de Oceanografia, Centro de Ciências Físicas e Matemáticas, Universidade Federal de Santa Catarina. E-mail: thalesvf@hotmail.com ${ }^{2}$ Laboratório de Oceanografia Costeira, Universidade Federal de Santa Catarina, bolsista de Produtividade em Pesquisa do CNPq, Coordenadoria Especial de Oceanografia, Centro de Ciências Físicas e Matemáticas, Universidade Federal de Santa Catarina. E-mail: jarbas.bonetti@ufsc.br
\end{abstract}

\section{RESUMO}

A repercussão dos efeitos de eventos extremos na zona costeira, sobretudo nos processos de erosão, tem estimulado a proposição de diferentes alternativas metodológicas para a análise de sua vulnerabilidade. A identificação das áreas mais suscetíveis à erosão representa um elemento de apoio à gestão e prevenção de desastres naturais, mas pouca atenção tem sido dada ao desenvolvimento de estratégias de mapeamento dos impactos efetivamente sofridos. Este estudo tem como objetivo propor uma metodologia para o mapeamento de danos baseada no emprego de indicadores de eventos erosivos na Praia dos Ingleses, SC. Após a ocorrência de fortes eventos erosivos na área de estudo, foram realizados levantamentos de campo para identificar feições pontuais que caracterizassem distintos estágios erosionais no pós-praia, tendo os mesmos servido de base para a elaboração de um esquema de classificação contínuo ao longo da área. O mapeamento dos níveis de danos mostrou que existe uma variabilidade importante na exposição da praia estudada, havendo uma relação direta entre a proximidade da urbanização à linha de costa e supressão de duna frontal com as maiores intensidades dos danos sofridos.

Palavras-chave: suscetibilidade, erosão costeira, análise espacial, geoindicadores, índices. 


\begin{abstract}
The repercussion of extreme events in the coastal zone, especially regarding erosion processes, has stimulated the proposition of different methodological approaches for vulnerability assessment. The identification of areas most susceptible to erosion may constitute an important guidance for the management and prevention of natural disasters. However, little attention has been given to the development of mapping strategies to assess effective undergone impacts. This study aims to propose a methodological framework for damage mapping based on the use of erosional indicators in Praia dos Ingleses, southern Brazil. After strong erosive events on the beach field surveys were performed to identify point features that could characterize different erosional levels in the backshore, allowing the elaboration of a classification scheme that could represent the hole beach. The resulting map of damage levels revealed that there is an important variation in the exposure of the studied beach. In the area, higher damage intensities were mostly related to the proximity to the sea of urban development and to the suppression of the original foredune.
\end{abstract}

Keywords: susceptibility, coastal erosion, spatial analysis, geoindicators, indices.

\title{
INTRODUÇÃO
}

A zona costeira constitui um importante espaço do planeta, onde se concentram as maiores cidades e grande parte da população mundial (Nicholls, 1995). No ano de 1990, $23 \%$ da população mundial já se assentava sobre áreas localizadas em até $100 \mathrm{~km}$ de distância da costa e em altitudes inferiores a 100 m acima do nível do mar, sendo a concentração populacional em setores costeiros aproximadamente o triplo da média global (Small \& Nicholls, 2003). No Brasil, 13 das 27 capitais estaduais estão situadas na zona costeira, entre elas metrópoles como Rio de Janeiro, Salvador, Recife, Fortaleza e Florianópolis (MMA, 2008).

Nesse contexto, a erosão costeira pode ser considerada um fenômeno global que afeta grande parte das zonas litorâneas de todo o mundo. Além de ser um sistema extremamente dinâmico e naturalmente suscetível a eventos de alta energia, a costa ainda está sujeita às ações antrópicas que interferem nos processos locais e intensificam os efeitos da erosão (Klein et al., 2016).

De maneira geral, a erosão costeira se caracteriza pela perda de sedimentos através de processos geomorfológicos, resultante da complexa interação entre ondas, nível do mar, correntes costeiras, balanço de sedimentos e geologia (Komar, 1983). Segundo Komar (1983), praias arenosas estão sujeitas à ação erosiva e acresciva durante eventos de alta energia e períodos de menor energia de ondas, respectivamente. Sendo assim, praticamente todas as praias apresentam suscetibilidade à erosão durante eventos de tempestades (Rudorff \& Bonetti, 2010).

No estado de Santa Catarina existe um significativo registro de desastres ambientais associados à erosão resultantes da ação combinada entre ondas grandes, ventos regulares e marés de sizígia (Rudorff et al., 2014). Um dos principais fatores dessa alta exposição decorre de sua localização transicional, que expõe a zona costeira à influência de sistemas meteorológicos e oceanográficos de alta energia. 
A Praia dos Ingleses, localizada no norte da Ilha de Santa Catarina (Figura 1), tem um considerável histórico relacionado a eventos extremos, sobretudo à ação de marés de tempestade que induzem à ocorrência de erosão. Essa praia já foi classificada por diversos autores como possuidora de um nível de suscetibilidade à erosão entre médio e muito alto (Rudorff \& Bonetti, 2010; Mussi, 2011; Muler, 2012; Furtado, 2018). Tal suscetibilidade, que é reflexo das características naturais do sistema, foi amplificada localmente devido à intensa urbanização, que em diversos trechos avança sobre o próprio prisma praial emerso. $\mathrm{O}$ efeito da intensificação da exposição natural de um sistema devido à interferência da infraestrutura instalada em seu processo de ocupação pode ser definido como "suscetibilidade induzida" (Bonetti et al., 2018).
Figura 1 - Localização da área de estudo

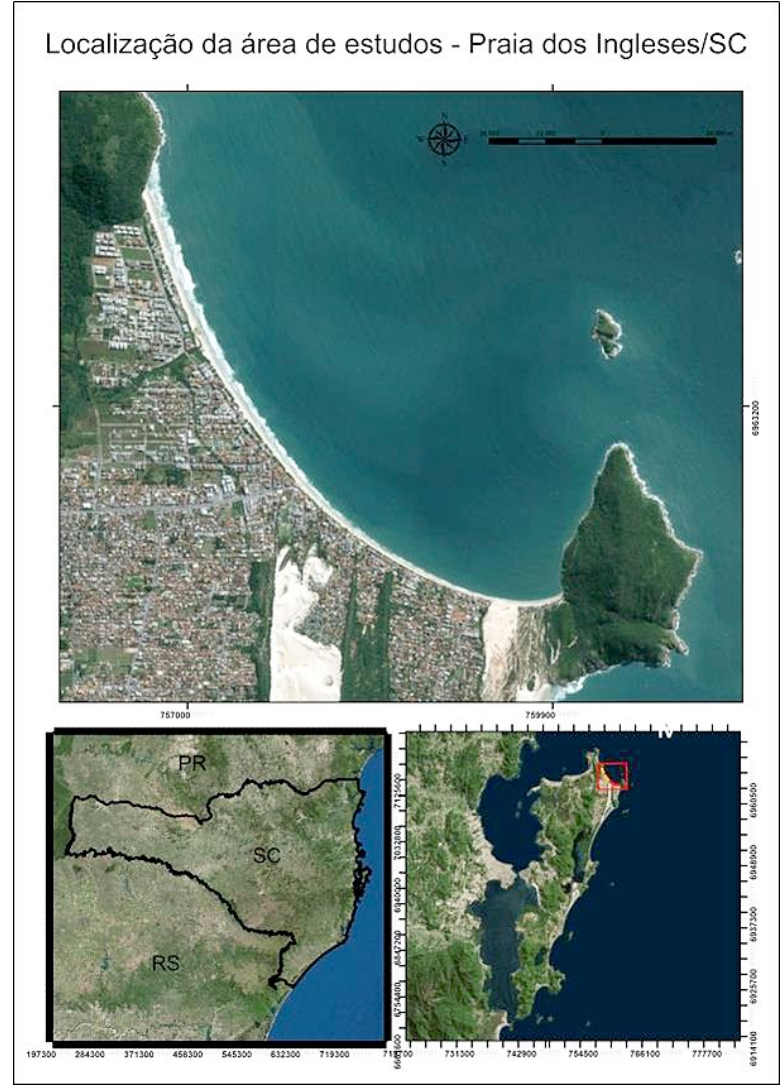

A maior repercussão dos impactos sobre a zona costeira, com significativos prejuízos econômicos, estimulou a formulação de diferentes metodologias para a identificação da suscetibilidade à erosão, sobretudo visando ao planejamento de seu uso e sua ocupação (Bonetti \& Woodroffe, 2017). Não obstante o largo emprego desses modelos, sobretudo aqueles baseados na aplicação de índices (Nguyen et al., 2016), até o momento pouca atenção foi dada ao desenvolvimento de estratégias de representação espacial dos impactos efetivamente sofridos pelos diferentes setores que compõem uma praia após a ocorrência de um evento extremo. Esses registros de danos podem constituir a verdade de campo para a avaliação do desempenho de modelos preditivos e, igualmente, podem se basear na aplicação de índices classificatórios. Nesse contexto, este estudo tem como objetivo propor uma metodologia para a avaliação de impactos com base em indicadores pontuais de danos causados por eventos extremos.

\section{MATERIAIS E MÉTODOS}

O levantamento dos danos sofridos ao longo do pós-praia foi realizado em duas atividades de campo voltadas à identificação e ao registro de indicadores de processos erosivos, sendo os dados obtidos integrados e processados em ambiente SIG. A primeira campanha ocorreu no dia 2 de junho de 2017, logo após o início de um forte evento erosivo que atingiu várias praias da Ilha de Santa Catarina, e a segunda no dia 16 de outubro, posteriormente a outros dois eventos de alta energia que instabilizaram ainda mais a praia. A reali- 
zação de duas campanhas permitiu não apenas identificar os locais mais afetados, mas também verificar a evolução dos danos a partir do primeiro evento.

Ao todo foram descritos 47

Figura 2 - Distribuição dos pontos descritos em campo, integrando as duas campanhas

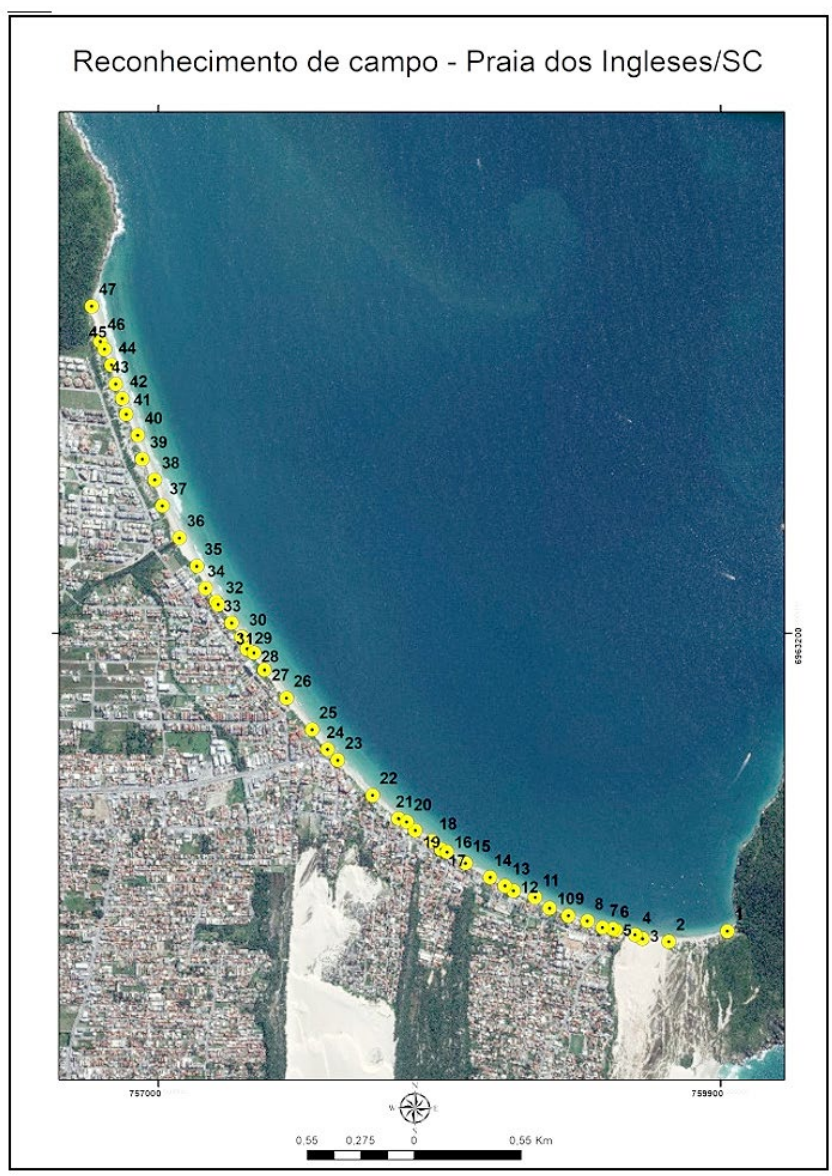

pontos ao longo de toda a praia, 26 no primeiro trabalho de campo, aos quais foram adicionados 21 novos pontos na segunda campanha (Figura 2). As coordenadas dos pontos foram determinadas com o auxílio de um GPS de navegação Garmin e de um GPS acoplado a uma câmera fotográfica Nikon Coolpix P510. As fotografias georreferenciadas possibilitaram o registro das feições geomorfológicas, a identificação da presença de construções e a determinação da intensidade dos danos causados em cada ponto ao longo da praia.

Para cada ponto amostrado avaliou-se visualmente as feições morfossedimentares indicativas de danos, que foram posteriormente tabuladas de acordo com a classificação do estágio erosional adaptada de Bush et al. (1999) e Bonetti et al. (2018). Com base na atribuição de pesos às feições descritas, foram obtidos valores de intensidade dos danos que ocorreram em cada campanha (Figura 3).

Figura 3 - Indicadores utilizados para a classificação do estado erosional das praias

\begin{tabular}{|c|c|c|c|c|c|}
\hline $\begin{array}{l}\text { Estado } \\
\text { Erosional }\end{array}$ & Peso & Indicadores / feições & $\begin{array}{l}\text { Números de } \\
\text { identificação }\end{array}$ & $\begin{array}{c}\text { Registro do evento } \\
(28 / 05 / 2017)\end{array}$ & $\begin{array}{c}\text { Registro Pós-Evento } \\
(06 / 10 / 2017)\end{array}$ \\
\hline \multirow{3}{*}{$\begin{array}{l}\text { Erosão } \\
\text { extrema }\end{array}$} & \multirow{3}{*}{3} & $\begin{array}{l}\text { Perda total ou parcial de } \\
\text { estruturas residenciais }\end{array}$ & 1.1 & & \\
\hline & & Ausência de praia seca; & 1.2 & & \\
\hline & & $\begin{array}{l}\text { Ausência por destruição de obras } \\
\text { de contenção. }\end{array}$ & 1.3 & & \\
\hline \multirow{3}{*}{$\begin{array}{l}\text { Erosão } \\
\text { severa }\end{array}$} & \multirow{3}{*}{2} & Presença de obras de proteção; & 2.1 & & \\
\hline & & $\begin{array}{l}\text { Danos a estruturas fisicas e } \\
\text { naturais; }\end{array}$ & 2.2 & & \\
\hline & & Raizes expostas; & 2.3 & & \\
\hline \multirow{3}{*}{$\begin{array}{l}\text { Erosão } \\
\text { leve }\end{array}$} & \multirow{3}{*}{1} & Dunas escarpadas; & 3.1 & & \\
\hline & & Transporte sedimentar visivel; & 3.2 & & \\
\hline & & $\begin{array}{ll}\text { Vegetação } & \text { parcialmente } \\
\text { danificada } & \end{array}$ & 3.3 & & \\
\hline
\end{tabular}


Nesta pesquisa foram adotadas três diferentes classes de danos erosivos: leves, severos e extremos, não ocorrendo trechos sem dano algum.

Os tipos de danos representaram feições observadas in situ. Como indicadores representativos da classe de danos leves, foram identificados escarpas em dunas, evidências de transporte sedimentar e vegetação levemente danificada. Para a classe com danos severos, foram descritas obras de proteção costeira, estruturas danificadas e vegetação com raízes expostas. Por fim, para a classe de danos extremos, observou-se a destruição total de infraestruturas, ausência de praia seca e eliminação, por destruição, de obras de proteção costeira.

Todos os pontos selecionados foram tabulados com seu respectivo número, identificação fotográfica, classe de nível de dano, código de identificação referente ao dano e peso da classe (Figura 3). O valor final de grau de dano foi obtido pela geração de um índice resultante da somatória do número de indicadores registrados em cada ponto e seus respectivos pesos. Os valores obtidos em cada ponto foram então classificados de acordo com o método de quebra natural (Natural Breaks) ou método de Jenks (Jenks \& Caspall, 1971).

O resultado foi transformado em linhas para melhor representação da distribuição dos danos ao longo da linha de costa. Essa transformação foi realizada manualmente no software ArcGis 10.1. Assim, foram gerados dois mapas de níveis de danos para a Praia dos Ingleses, um referente a cada campanha realizada.

\section{RESULTADOS}

Os danos observados foram significativos ao longo de toda praia e em ambas as campanhas, porém com maior intensidade nas proximidades das áreas mais ubanizadas, ao sul da praia (Figuras 4 e 5). Esse resultado é concordante com Klein et al. (2016), para quem toda a praia se encontra em estágio de erosão, não existindo acresção em nenhum setor.

Os pontos classificados como com "erosão extrema" refletiram os danos diretos a estruturas antrópicas, enquanto aqueles associados à classe "erosão leve" estiveram mais relacionados a danos aos ecossistemas naturais. É importante salientar, entretanto, que a presença de estruturas de contenção, por vezes, "camuflaram" o real estado erosional devido ao seu elevado grau de adaptação às condições erosionais recorrentes no local.

Da campanha 1 para a 2 notou-se um aumento perceptível de es-
Figura 4-Mapeamento da intensidade de danos da primeira campanha

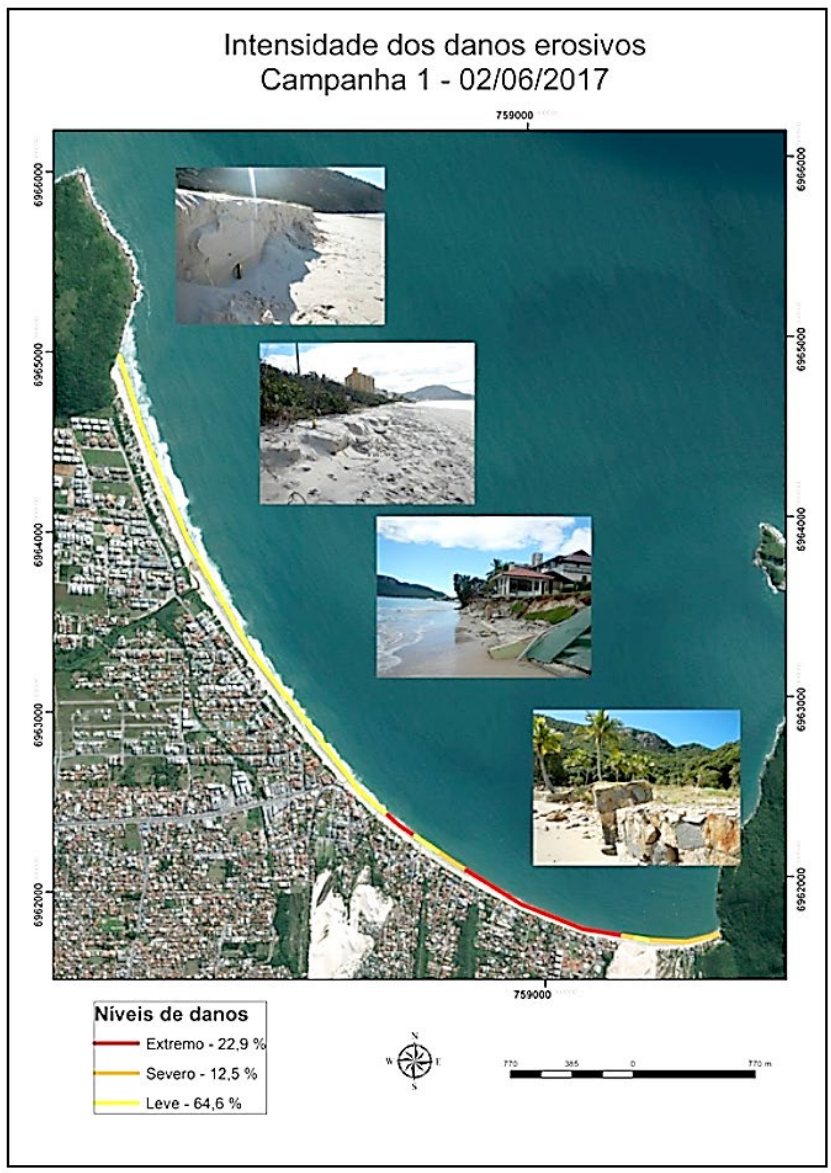


truturas danificadas, representadas pelos setores classificados como "erosão severa", áreas com estruturas antrópicas que não haviam sido atingidas no primeiro evento. Em contrapartida, houve diminuição de pontos pertencentes à classe "erosão extrema", apesar de alguns setores que não se enquadravam nessa classe terem passado a apresentar danos erosivos de grande intensidade.

\section{DISCUSSÃO}

O mapeamento dos danos permitiu destacar áreas mais afetadas, importante para uma adequada avaliação da suscetibilidade costeira local, tanto natural quanto induzida, como também prevenir futuros impactos através da gestão das áreas consideradas críticas.

No mapeamento gerado para as duas datas distintas percebeu-se a evolução dos danos na praia no decorrer dos eventos erosivos. Como foram eventos ocorridos sucessivamente em menos de três meses de intervalo, não houve tempo suficiente para o sistema se restabelecer, o que incorreu no aumento das áreas afetadas. Não foram levantados dados sobre volume e largura de praia, mas foi perceptível a redução significativa que houve ao longo desses meses.

De maneira geral, através da análise espacial dos dois mapas gerados, percebe-se claramente que o setor sul da praia, onde há maior adensamento de ocupação urbana próximo à linha d'água e sobre antigas dunas frontais, representa o trecho com maiores danos a estruturas antrópicas, associado à classe de danos extremos. Essa ocupação influencia diretamente o balanço sedimentar local, fazendo com que haja perda de sedimentos durante eventos erosivos sem reposição posterior, uma vez que estes são levados para além da zona de surfe.

É interessante notar que esse setor da praia foi ocupado originalmente pela comunidade por ser o local mais abrigado da ação de ondas ao longo do arco praial, protegido das ondulações de sul, que são as de maior energia na Ilha de Santa Catarina (Muler \& Bonetti, 2014). Porém, o local não está resguardado de ondulações das direções leste e nordeste, menos frequentes e de menor intensidade. A ocupação intensa sobre as dunas afetou o balanço sedimentar, gerando uma suscetibilidade induzida no local.

No levantamento de campo realizado, em ambas as datas, a classe predominante foi a relacionada a danos erosivos leves, por não afetarem estruturas físicas antrópicas, como 
casas e restaurantes. Todavia, o dano ao ecossistema foi visível, apesar de a praia ainda apresentar área significativa com presença de dunas e vegetação de restinga no pós-praia, principalmente do centro para o norte.

Embora visualmente menos impactante, a classe erosão leve, predominante no norte da praia, foi relevante e pode representar perdas significativas de serviços ecossistêmicos. Avaliando-se os resultados obtidos ao longo de toda a praia, pode-se supor que, caso houvesse ocupações nessas áreas, elas estariam igualmente comprometidas e sofreriam danos graves. Dessa forma, não obstante a presença de uma suscetibilidade natural da praia à erosão, conforme atestado por outros autores (Rudorff \& Bonetti, 2010; Mussi; Bonetti \& Sperb, 2018), a componente de exposição aos eventos extremos induzida pela presença antrópica parece ter sido determinante na distribuição dos impactos sofridos ao longo do arco praial.

A comparação dos resultados obtidos em ambas as campanhas indica o aumento da classe de danos severos, principalmente no setor central do arco praial, e a evolução de algumas áreas classificadas como danos severos para extremos, porém com uma leve redução percentual da classe de danos extremos. Isso se deve principalmente à capacidade de recuperação da população atingida, uma vez que algumas áreas impactadas no primeiro evento já haviam sido recuperadas e protegidas.

Tal fato chama a atenção para a importância, nos estudos sobre vulnerabilidade costeira, de se considerar também a capacidade adaptativa das populações, que podem fazer frente aos danos até um determinado grau e contribuir para a diminuição da vulnerabilidade local (Serafim \& Bonetti, 2017; Serafim et al., 2019).

A metodologia para avaliação dos danos se mostrou satisfatória, uma vez que representou adequadamente áreas mais afetadas durante eventos erosivos na praia. Propõe-se que seja utilizada como elemento de apoio à validação de modelos espaciais de vulnerabilidade ou suscetibilidade costeira, podendo ser empregada como elemento de aferição de modelos preditivos.

\section{REFERÊNCIAS BIBLIOGRÁFICAS}

Bonetti, J. \& Woodroffe, C.D. Spatial analysis on GIS for coastal vulnerability assessment, p. 367-396, in Bartlett, D. \& Celliers, L. (eds.), Geoinformatics for marine and coastal management, Boca Raton: CRC Press, 414 p., 2017.

Bonetti, J.; Rudorff, F.M.; Campos, A.V. \& Serafim, M.B. Geoindicator-based assessment of Santa Catarina (Brazil) sandy beaches susceptibility to erosion. SI: Management Strategies for Coastal Erosion Problems. Ocean E Coastal Management, v. 156, p. 198-208, 2018.

Bush, D.M.; Neal, W.J.; Young, R.S. \& Pilkey, O.H. Utilization of geoindicators for rapid assessment of coastal hazard risk and mitigation. Ocean $\mathcal{E}$ Coastal Management, v. 42, p. 647670, 1999.

Furtado, T.V. Avaliação de modelos espaciais de vulnerabilidade costeira à erosão: eventos recentes na Praia dos Ingleses - SC. Dissertação de mestrado, Programa de Pós-Graduação em Geografia, Universidade Federal de Santa Catarina, 113 p., Florianópolis, 2018.

Jenks, G.F. \& Caspall F.C. Error on choroplethic maps: definition, measurement, reduction. Annals of the Association of American Geographers, v. 61, n. 2, p. 217-244, 1971. 
Klein, A.H.F.; Prado, M.F.V.; Dalinghaus, C. \& Camargo, J.M. Metodologia para quantificação de perigos costeiros e projeção de linhas de costa futuras como subsídio para estudos de adaptação das zonas costeiras: litoral norte da Ilha de Santa Catarina e entorno. Brasília: Ministério do Meio Ambiente, 251 p., 2016.

Komar, P.D. Handbook of coastal processes and erosion. Florida: CRC Press, 316 p., 1983.

MMA. Macrodiagnóstico da zona costeira e marinha do Brasil. Brasília: Ministério do Meio Ambiente, 242 p., 2008.

Muler, M. Avaliação da vulnerabilidade de praias da Ilha de Santa Catarina a perigos costeiros através da aplicação de um índice multicritério. Dissertação de mestrado, Programa de Pós-Graduação em Geografia, Universidade Federal de Santa Catarina, 189 p., Florianópolis, 2012.

Muler, M. \& Bonetti, J. An integrated approach to assess wave exposure in coastal areas for vulnerability analysis. Marine Geodesy, v. 37, n. 2, p. 220-237, 2014.

Mussi, C.S. Avaliação da sensibilidade ambiental costeira e de risco à elevação média dos oceanos e incidência de ondas de tempestade: um estudo de caso para Ilha de Santa Catarina, SC. Dissertação de mestrado, Programa de Pós-Graduação em Ciência e Tecnologia Ambiental, Universidade do Vale do Itajaí, 128 p., Itajaí, 2011.

Mussi, C.S.; Bonetti, J. \& Sperb, R.M. Coastal sensitivity and population exposure to sea level rise: a case study on Santa Catarina Island, Brazil. Journal of Coastal Conservation, v. 22, n. 6, p. 1117-1128, 2018.

Nguyen, T.T.X.; Bonetti, J.; Rogers, K. \& Woodroffe, C.D. Indicator-based assessment of climate-change impacts on coasts: a review of concepts, approaches and vulnerability indices. Ocean \& Coastal Management, v. 123, p. 18-43, 2016.

Nicholls, R.J. Coastal megacities and climate change. GeoJournal, v. 37, n. 3, p. 369-379, 1995.

Rudorff, F.M. \& Bonetti, J. Avaliação da suscetibilidade à erosão costeira de praias da Ilha de Santa Catarina com base em geoindicadores e técnicas de análise espacial de dados. Brazilian Journal of Aquatic Science and Technology, v. 14, p. 9-20, 2010.

Rudorff, F.M.; Bonetti, J.; Moreno, D.A.; Oliveira, C.A.F. \& Murara, P.G. Maré de tempestade, p. 151-154, in Herrmann, M.L.P. (ed.), Atlas de desastres naturais do estado de Santa Catarina: período de 1980 a 2010, 2. ed., Florianópolis: IHGSC/Cadernos Geográficos, 217 p., 2014.

Serafim, M. \& Bonetti. J. Vulnerabilidade das praias do estado de Santa Catarina a eventos de erosão e inundação costeira: proposta metodológica baseada em um índice multicritério. Quaternary and Environmental Geosciences, v. 8, n. 2, p. 36-54, 2017.

Serafim, M.B.; Siegle, E.; Corsi, A.C. \& Bonetti, J. Coastal vulnerability to wave impacts using a multi-criteria index: Santa Catarina (Brazil). Journal of Environmental Management, v. 230, p. 21-32, 2019.

Small, C. \& Nicholls, R.J.A. Global analysis of human settlement in coastal zones. Journal of Coastal Research, v. 19, n. 3, p. 584-599, 2003. 
